ARTICLE

Received 31 Jan 2014 | Accepted 17 Apr 2014 | Published 23 May $2014 \quad$ DOl: 10.1038/ncomms4910

\title{
Chiral spin torque arising from proximity-induced magnetization
}

Kwang-Su Ryu ${ }^{1}$, See-Hun Yang ${ }^{1}$, Luc Thomas ${ }^{1, \dagger} \&$ Stuart S.P. Parkin ${ }^{1}$

Domain walls can be driven by current at very high speeds in nanowires formed from ultra-thin, perpendicularly magnetized cobalt layers and cobalt/nickel multilayers deposited on platinum underlayers due to a chiral spin torque. An important feature of this torque is a magnetic chiral exchange field that each domain wall senses and that can be measured by the applied magnetic field amplitude along the nanowire where the domain walls stop moving irrespective of the magnitude of the current. Here we show that this torque is manifested when the magnetic layer is interfaced with metals that display a large proximity-induced magnetization, including iridium, palladium and platinum but not gold. A correlation between the strength of the chiral spin torque and the proximity-induced magnetic moment is demonstrated by interface engineering using atomically thin dusting layers. High domain velocities are found where there are large proximity-induced magnetizations in the interfaced metal layers.

\footnotetext{
${ }^{1}$ IBM Almaden Research Center, 650 Harry Road, San Jose, California 95120, USA. † Present address: TDK-Headway Technologies, Milpitas, California, USA Correspondence and requests for materials should be addressed to S.S.P.P. (email: stuart.parkin@icloud.com).
} 
T he manipulation of domain walls by spin-polarized currents allows for novel memory ${ }^{1,2}$ and logic ${ }^{3}$ devices that were not previously possible by using magnetic fields alone. Early studies on the current-driven motion of domain walls (DWs) in both in-plane $e^{4-8}$ and thick, perpendicularly magnetized $\mathrm{Co} / \mathrm{Ni}$ nanowires ${ }^{9}$ could be well understood by the phenomenon of volume spin-transfer torque (V-STT) ${ }^{10-12}$ that arises from the transfer of spin angular momentum from spin-polarized current in the interior of the magnetic material to the local magnetization ${ }^{10,11}$. Much higher DW velocities were subsequently reported in ultrathin ferromagnetic layers displaying large perpendicular magnetic anisotropy (PMA) $)^{13-15}$ that are difficult to reconcile with V-STT. Furthermore, the DWs move in the direction of current, opposite to that expected from conventional V-STT ${ }^{13-16}$. Two mechanisms that were initially proposed to account for this unexpected behaviour, based on Rashba magnetic fields at magnetic/oxide interfaces ${ }^{17-19}$, and on spin accumulation derived from spin currents generated through
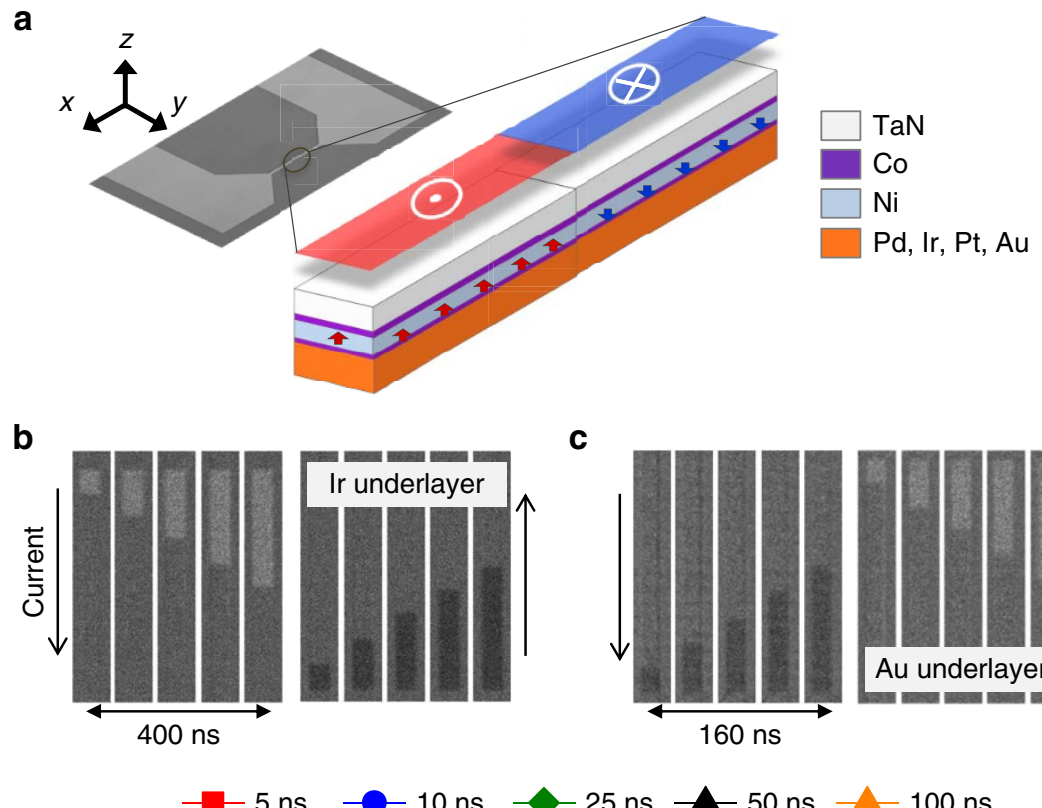

C
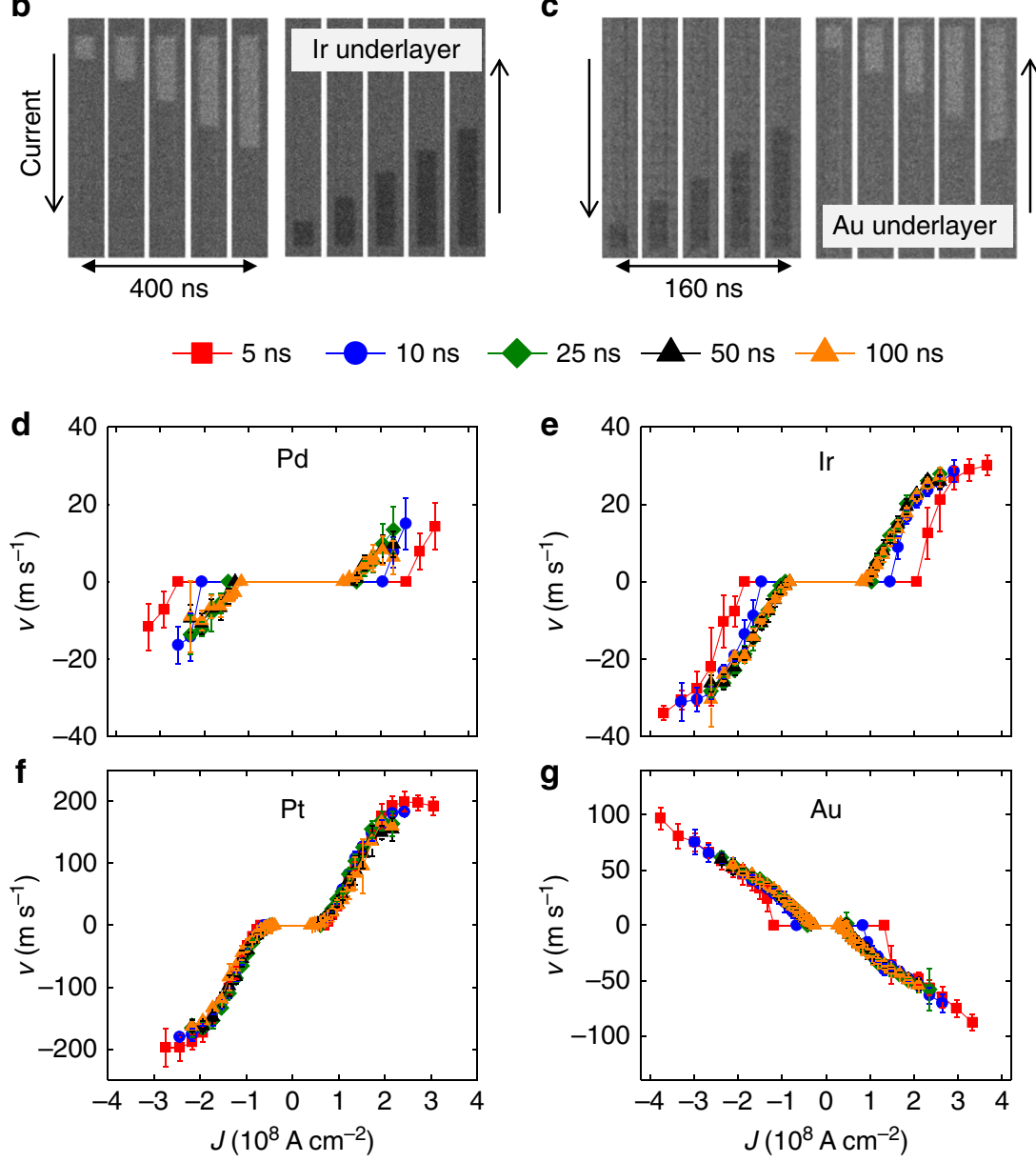

Figure 1 | Dependence of current-driven DW motion in Co/Ni wires on underlayer. (a) Schematic representation of a device showing a UL/ 3 Co/7 $\mathrm{Ni} / 1.5$ Co stack grown on various ULs and two magnetic domains in which the magnetization is perpendicular to the plane of the wafer: red and blue correspond to magnetization pointing out of or into the plane, respectively. (b,c) Kerr microscopy images of a single DW moving along a nanowire in response to sequences of current pulses $\left(J \sim 3.0 \times 10^{8} \mathrm{~A} \mathrm{~cm}^{-2}\right)$ composed of ten 10-ns and eight 5-ns long pulses. Typical images are shown for Ir and Au ULs, respectively, for positive and negative current polarities, as indicated by the arrows. Note that the lighter and darker contrast corresponds to up and down magnetization directions, respectively. (d-g) DW velocities as a function of the current pulse density J for 50 Pd, $30 \mathrm{lr}, 50 \mathrm{Pt}$ and $30 \mathrm{Au}$ ULs. The current pulse lengths are varied from $5 \mathrm{~ns}$ (red squares), $10 \mathrm{~ns}$ (blue circles), $25 \mathrm{~ns}$ (green diamonds), $50 \mathrm{~ns}$ (black triangles), to $100 \mathrm{~ns}$ (orange triangles), respectively. The error bars in all figures correspond to one s.d. All thicknesses are given in $\AA$. 
the spin Hall effect (SHE) ${ }^{20,21}$ in platinum underlayers (ULs) ${ }^{22}$, cannot alone account for the observed current induced motion of the DWs in PMA nanowires ${ }^{15}$.

Recently, it has been shown experimentally that in perpendicularly magnetized $\mathrm{Co} / \mathrm{Ni} / \mathrm{Co}$ stacks grown on Pt layers, in which the DWs move very fast, the DWs are subject to large internal effective fields directed along the nanowire, which alternate in direction between successive DWs ${ }^{15}$. These internal fields cause the most stable DW structure to be a Néel structure, in which the magnetization rotates in a plane parallel to the length of the nanowire, rather than a Bloch structure, and, moreover, establish that all the DWs in the wire have the same chirality.
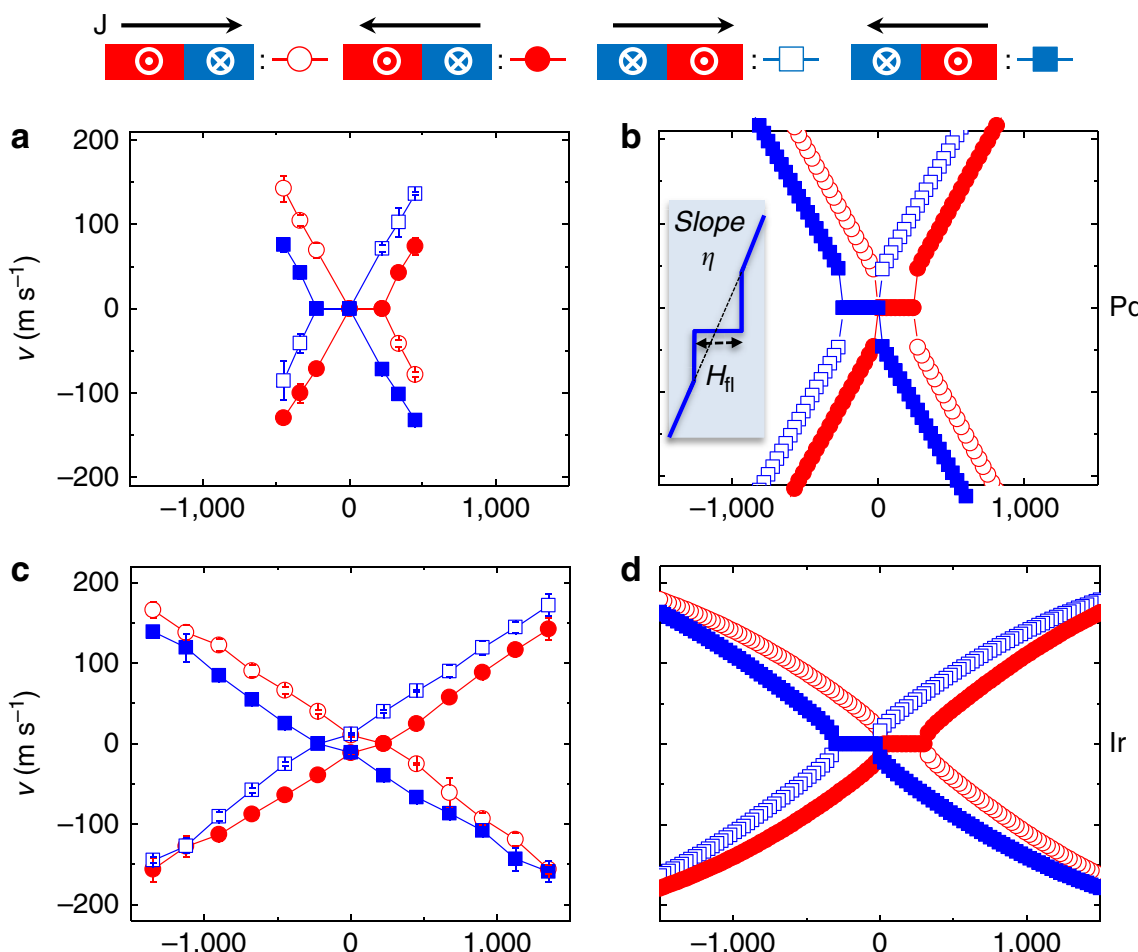

d
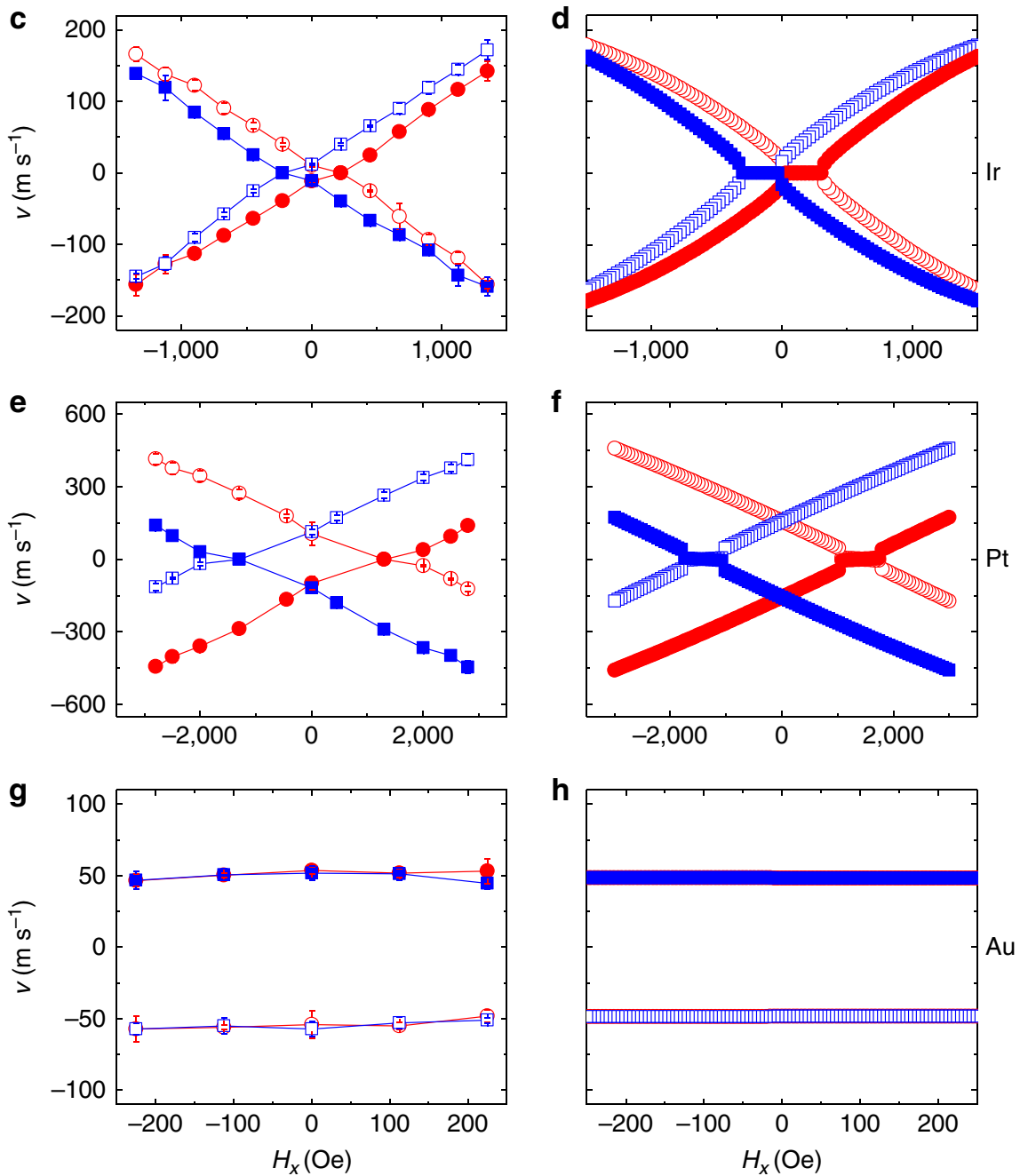

Figure 2 | Dependence of current-driven DW dynamics on underlayer. (a,c,e,g) Measured DW velocity versus $H_{x}$ and $(\mathbf{b}, \mathbf{d}, \mathbf{f}, \mathbf{h})$ corresponding 1D model calculations for UL/ $3 \mathrm{Co} / 7 \mathrm{Ni} / 1.5 \mathrm{Co}$ wires with $50 \mathrm{Pd}, 30 \mathrm{lr}, 15 \mathrm{Pt}$ and $30 \mathrm{Au}$ ULs, respectively for $\mathrm{J} \sim 2 \times 10^{8} \mathrm{Acm} \mathrm{cm}^{-2}$. Red and blue symbols represent $\uparrow \downarrow$ and $\uparrow \downarrow D W s$, respectively. Open and solid symbols correspond to positive $(+x)$ and negative $(-x)$ current directions, respectively. Key fitting parameters used in the 1D model are summarized along with key experimentally determined parameters in Table 1. (See details in Supplementary Table 1). The error bars in $(\mathbf{a}, \mathbf{c}, \mathbf{e}, \mathbf{g})$ correspond to one s.d. All thicknesses are in $\AA$. The inset in $\mathbf{b}$ shows the definitions of $\eta$ and $H_{\mathrm{fl}}$. 
The combined effect of these internal fields together with SHE-generated spin currents gives rise to a chiral spin torque (CST) that is responsible for the high velocity of DWs along the current direction in $\mathrm{Pt} / \mathrm{Co} / \mathrm{Ni} / \mathrm{Co}$ nanowires ${ }^{15,23-25}$.

The origin of the internal fields responsible for the CST has been speculated to be derived from a Dyzaloshinskii-Moriya interaction $(\mathrm{DMI})^{15,26-31}$ at the $\mathrm{Pt} / \mathrm{Co}$ interface. However, the DMI originates from spin-orbit coupling (SOC) that is large in $\mathrm{Pt}$ but much weaker in $\mathrm{Co}$ and $\mathrm{Ni}$, and the mechanism by which the DMI can generate such large internal fields at the DWs in the $\mathrm{Co} / \mathrm{Ni} / \mathrm{Co}$ magnetic trilayer is not understood.

Here we show that the CST is not limited to Pt ULs but is found for other heavy metal ULs including Pd and Ir, but not Au. We find that the strength of the internal effective fields in these different systems is consistent with their originating from a DMI. Moreover, we show that the CST is only observed when the magnetic trilayer is in contact with metals that display a proximity-induced magnetic moment (PIM), thus shedding new light on the mechanism underlying the CST in these systems.

\section{Results}

Underlayer dependence of current induced domain wall motion. Nanowires were formed from magnetic sandwiches of ( $3 \AA \mathrm{Co} / 7 \AA \mathrm{Ni} / 1.5 \AA \mathrm{Co}$ ) deposited on metallic ULs of Pd, Ir, Pt and $\mathrm{Au}$. Only structures that exhibited perpendicular magnetic anisotropy (PMA) with square magnetic hysteresis loops were used for these studies. The current-induced motion of individual DWs was studied in nanowires $2 \mu \mathrm{m}$ wide and $50 \mu \mathrm{m}$ long (Fig. 1a) that were fabricated by optical lithography and argon ion mill etching. Kerr optical microscopy (see Methods for details) was used to track the motion of individual domain walls along the nanowires in response to nanosecond long current pulses. Typical examples of Kerr images that were used to determine the direction and velocity of the DW motion in $\mathrm{Co} / \mathrm{Ni} / \mathrm{Co}$ nanowires on Ir and Au ULs, respectively, are shown in Fig. 1b,c. The dependence of DW velocity on the current density $J$ is shown in Fig. $1 \mathrm{~d}-\mathrm{g}$ for $[\mathrm{Co} / \mathrm{Ni} / \mathrm{Co}]$ nanowires grown on $\mathrm{Pd}, \mathrm{Ir}, \mathrm{Pt}$ and $\mathrm{Au}$, respectively, for current pulses varying in length from 5 to $100 \mathrm{~ns}$. In each case, the DWs only move above a threshold current density $J_{\mathrm{C}}$ that increases the shorter is the current pulse length. $J_{\mathrm{C}}$ varies significantly with the UL metal, as does the maximum DW velocity, which is much smaller for Pd and Ir than for Pt. For Pd, Ir and Pt ULs the DWs move in the direction of the current flow, whereas, by contrast, for ULs formed from $\mathrm{Au}$ the DWs move in the opposite direction along the electron flow. For all four metal ULs, however, all the DWs, whether up/down ( $\downarrow$ ) or down/up $(\downarrow \uparrow)$, move at the same speed and in the same direction.

Influence of longitudinal field on domain wall velocity. To explore the origin of the CST, we compare the dependence of the DW velocity $v$ (measured at $J=2 \times 10^{8} \mathrm{~A} \mathrm{~cm}^{-2}$ ) on a longitudinal magnetic field $H_{x}$ applied along the nanowire for $\mathrm{Co} / \mathrm{Ni} / \mathrm{Co}$ sandwiches prepared on $\mathrm{Pd}, \mathrm{Ir}, \mathrm{Pt}$ and $\mathrm{Au}$ ULs in Fig. 2a,c,e,g. Data are shown for both $\uparrow \downarrow$ and $\downarrow \uparrow$ DWs (red and blue symbols, respectively) and for positive and negative currents (open and closed symbols, respectively). For Pd, Ir and Pt ULs the dependence of $v$ on $H_{x}$ has two important characteristics. First, $v$ increases almost linearly with $H_{x}$ except for an offset close to $H_{x} \sim 0$ that is more clearly visible for the samples with Pd and Ir ULs. Second, the DW velocity becomes zero at a critical value of $H_{x}$ that we will name the crossing field, $H_{\mathrm{CR}}$. $H_{\mathrm{CR}}$ has the same magnitude but the opposite sign for $\uparrow \downarrow$ and $\downarrow \uparrow$ DWs. The behaviour for $\mathrm{Pd}$, Ir and $\mathrm{Pt}$ is similar to that which we reported previously for various $\mathrm{Co} / \mathrm{Ni} / \mathrm{Co}$ stacks grown on $\mathrm{Pt}^{15}$. In contrast, the data shown in Fig. $2 \mathrm{~g}$ for a Au UL are clearly very different and there is almost no dependence of $v$ on $H_{x}$. Note that the maximum value of $H_{x}$ that can be accessed is limited by the threshold field above which DWs are nucleated that is proportional to $K_{\mathrm{eff}}=K-2 \pi M_{\mathrm{S}}^{2}$, where $K$ is the perpendicular magnetic anisotropy constant and $M_{\mathrm{S}}$ is the saturation magnetization: thus, for $\mathrm{Au}$, and to a lesser extent Pd ULs, significantly smaller values of $H_{x}$ can be applied than for Pt and Ir ULs.

The dependence of $v$ on $H_{x}$ shown in Fig. 2a,c,e,g can be well understood within the 1D model (see Supplementary Note 1 and Supplementary Fig. 1), using the fitting parameters given in Table 1. As shown in the schematic diagram in the inset to Fig. $2 \mathrm{~b}$, there is a range of $H_{x}$ over which $v$ varies approximately linearly with $H_{x}$ with a slope that is given by $\eta=\mp \frac{\gamma \pi \Delta}{2} \frac{\frac{\pi}{2} H_{\mathrm{SHE}}}{\frac{\pi}{2} H_{\mathrm{SHE}}+\alpha H_{k}}$ where $\gamma$ is the gyromagnetic ratio, $\Delta$ is the DW width, $H_{\mathrm{SHE}}$ is the effective field derived from the SHE, $\alpha$ is the Gilbert damping

Table 1 | Summary of film magnetic properties and 1D model fitting parameters.

\begin{tabular}{|c|c|c|c|c|}
\hline$M_{\mathrm{S}}\left(\mathrm{emu} \mathrm{cm} \mathrm{cm}^{-3}\right)$ & 540 & 520 & 590 & 400 \\
\hline$\delta M_{\mathrm{S}}\left(\mathrm{emu} \mathrm{cm}^{-3}\right)$ & 120 & 100 & 170 & 0 \\
\hline$K\left(10^{6} \mathrm{erg} \mathrm{cm}^{-3}\right)$ & 2.5 & 3.0 & 5.4 & 1.9 \\
\hline$K_{\text {eff }}\left(10^{6} \mathrm{erg} \mathrm{cm}^{-3}\right)$ & 0.7 & 1.3 & 3.2 & 0.9 \\
\hline$H_{\mathrm{CR}}(\mathrm{Oe})$ & 120 & 180 & 1,400 & NA \\
\hline \multicolumn{5}{|l|}{ 1D model Parameters } \\
\hline$\Delta(\mathrm{nm})$ & 12 & 6.9 & 4.3 & 8.5 \\
\hline$\alpha$ & 0.04 & 0.07 & 0.10 & 0.10 \\
\hline$\beta$ & 0 & 0 & 0 & 0.08 \\
\hline$H_{\mathrm{SHE}}(\mathrm{Oe})$ & 550 & 100 & 1,000 & 0 \\
\hline$\theta_{\mathrm{SHE}}$ & 0.045 & 0.008 & 0.098 & 0 \\
\hline
\end{tabular}

Saturation magnetization, $M_{\mathrm{S}}$, perpendicular magnetic anisotropy constant, $K$ and $K_{\text {eff }}=K-2 \pi M_{S}^{2}$ are deduced from perpendicular and in-plane magnetic field dependences of the magnetic moment of the various films. $\delta M_{\mathrm{S}}$ is derived from the change in film magnetization when Au dusting layers are inserted at the UL/Co interface, as shown in Fig. $3 \mathrm{~b}$. The parameters, $\Delta, \alpha, \beta$ and $H_{\mathrm{SHE}}$, used in the $1 \mathrm{D}$ model fits shown in Fig. $2 b, \mathrm{~d}, \mathrm{f}, \mathrm{h}$ are listed in the Table. $H_{k}$ and $\theta_{\mathrm{SHE}}$ are calculated from experimental and fitted parameters, as discussed in the text. 

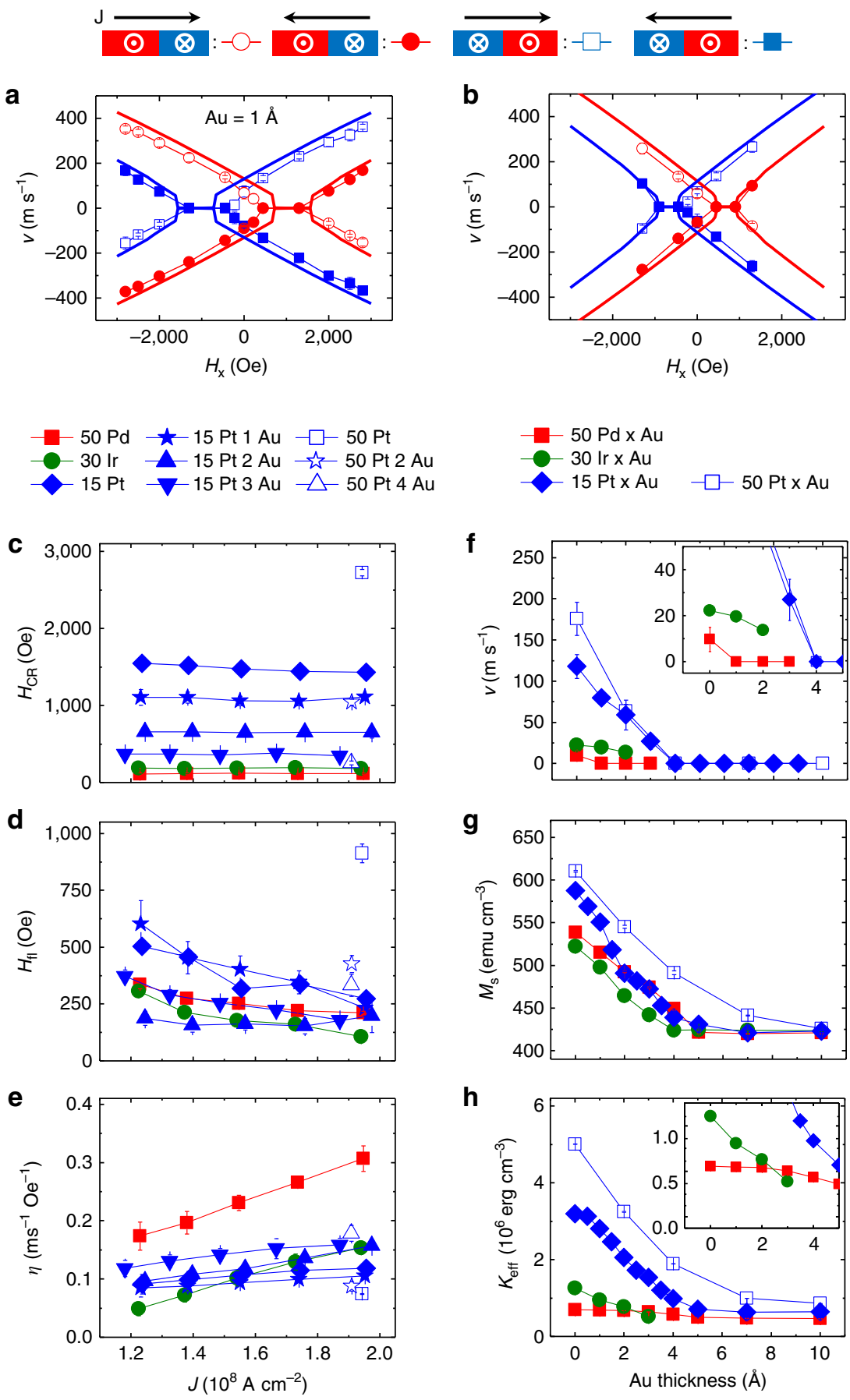

Figure 3 | Role of proximity-induced magnetization in underlayer. An ultra-thin Au spacer layer is inserted between the UL (50 Pd, $30 \mathrm{lr}$, $50 \mathrm{Pt}$, and $15 \mathrm{Pt}$ ) and the Co/Ni/Co stack. (a,b) Measured DW velocities versus $H_{x}$ for $15 \mathrm{Pt} / 1 \mathrm{Au}$ and $15 \mathrm{Pt} / 2 \mathrm{Au}$ ULs, respectively $\left(J \sim 2 \times 10^{8} \mathrm{Acm}{ }^{-2}\right)$. Red and blue symbols represent $\uparrow \downarrow$ and $\downarrow \uparrow D W s$, respectively. Open and solid symbols correspond to positive and negative currents. The 1D model fitted curves are plotted as solid lines. The error bars correspond to one s.d. (c-e), $H_{\mathrm{CR}}, H_{\mathrm{fl}}$ and $\eta$ versus J; $50 \mathrm{Pd}$ (red square), $30 \mathrm{Ir}$ (green circle), $15 \mathrm{Pt}$ (blue diamond), $15 \mathrm{Pt} / 1 \mathrm{Au}$ (blue star), $15 \mathrm{Pt} / 2 \mathrm{Au}$ (blue up triangle), $15 \mathrm{Pt} / 3 \mathrm{Au}$ (blue down triangle), $50 \mathrm{Pt}$ (blue open square), $50 \mathrm{Pt} / 2 \mathrm{Au}$ (blue open star) and $50 \mathrm{Pt} / 4 \mathrm{Au}$ (blue open triangle). The error bars in $\mathbf{c}, \mathbf{d}$ are s.e. values from the least square fits, while those in $\mathbf{e}$ correspond to one s.d. (f-h) Dependence of $v, M_{\mathrm{S}}$ and $K_{\text {eff }}$ on Au layer thickness $\left(J \sim 2 \times 10^{8} \mathrm{Acm}{ }^{-2}\right): M_{\mathrm{S}}$ and $K_{\text {eff }}$ are obtained from magnetization measurements on unpatterned films: $50 \mathrm{Pd} / x \mathrm{Au}$ (red squares), $30 \mathrm{lr} / x \mathrm{Au}$ (green circles), $15 \mathrm{Pt} / x \mathrm{Au}$ (solid blue diamonds) and $50 \mathrm{Pt} / \mathrm{x}$ Au (open blue squares). The error bars correspond to one s.d. All thicknesses are in $\AA$. For clarity the insets in $\mathbf{f}$ and $\mathbf{h}$ show an expanded view of the low $x$ data.

parameter and $H_{k}$ is the DW in-plane shape anisotropy field $\left(H_{k} \simeq 4 \pi M_{\mathrm{s}} \frac{t}{\Delta+t}\right.$ (ref. 32), where $t$ is the ferromagnetic layer thickness). Note that $H_{k}$ favours a Bloch DW for the wires used here. The sign of $\eta$ depends on the DW configuration ( - for $\uparrow \downarrow$ and + for $\downarrow \uparrow)$. The magnitude of $\eta$ varies in the order,
$\mathrm{Pd}>\mathrm{Ir}>\mathrm{Pt}$, largely due to the variation in $\Delta\left(\Delta \propto 1 / \sqrt{K_{\mathrm{eff}}}\right)$ that varies in the inverse order, $\mathrm{Pd}>\mathrm{Ir}>\mathrm{Pt}$ (see Table 1). Note that $\eta$ depends only weakly on $H_{\mathrm{SHE}}$. Near $H_{x}=H_{\mathrm{CR}}$ there is a discontinuity in the variation of $v$ with $H_{x}$ that may be accounted for by including in our $1 \mathrm{D}$ model static pinning of the DW 


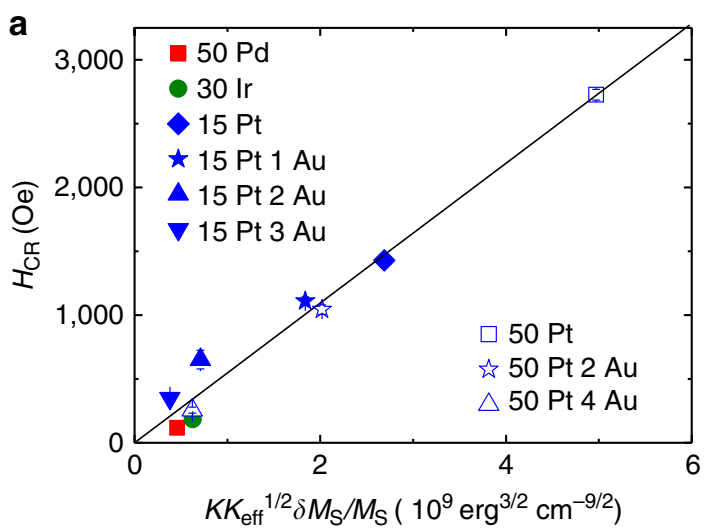

b

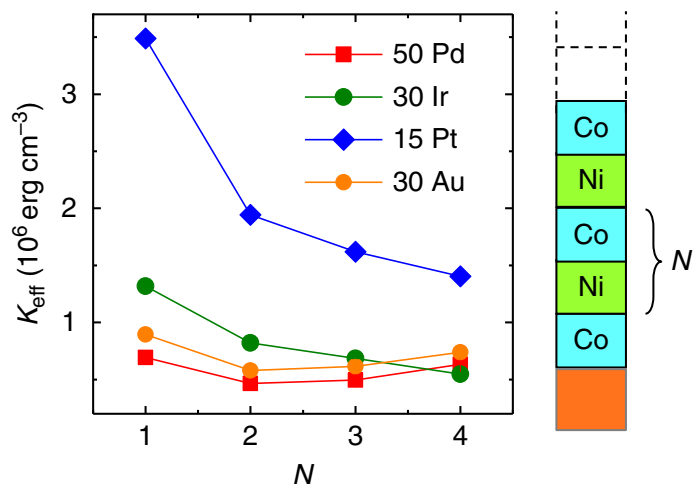

Figure 4 | Correlation between DMI and perpendicular anisotropy/ induced moment. (a) The crossing field $H_{C R}$ is plotted versus $K K_{\text {eff }}^{1 / 2} \delta M s / M s$ for various ULs. The black solid line is a linear fit to the data that is constrained to pass through zero. Here, the error bars correspond to those for $H_{C R}$. (b) $K_{\text {eff }}$ as a function of $\mathrm{N}$ for $[\mathrm{Co} / \mathrm{Ni}]_{\mathrm{N}}$ on various ULs, where $\mathrm{N}$ is varied from 1 to 4 . Error bars correspond to one s.d. All thicknesses are given in $\AA$.

(see Supplementary Note 1 and Supplementary Fig. 2). We define a window in $H_{x}$, centred around $H_{\mathrm{CR}}$ of width $H_{\mathrm{fl}}$ for which we find the DW velocity is zero, given by $\left|H_{x}-H_{\mathrm{CR}}\right| \leq H_{\mathrm{fl}} / 2$ (see inset to Fig. 2b).

The spin Hall angle $\theta_{\mathrm{SHE}}$ can be derived from $H_{\mathrm{SHE}}$, as discussed in the Supplementary Note 1 . As shown in Table 1, Pt displays the largest value of $\theta_{\text {SHE }}$ which is about two times that exhibited by Pd. $\theta_{\mathrm{SHE}}$ in Ir is much smaller. These values are in reasonable agreement with experimental results $22,33,34$ and theoretical predictions ${ }^{35}$. The data for Au ULs clearly show that there is no SHE for Au ULs and consequently no CST in this case.

Influence of Au dusting layers on domain wall dynamics. The strong dependence of the DW velocity on $H_{x}$ not only reflects the important role of the SHE but, most importantly, the crossing field where the DW velocity goes to zero is a direct manifestation of an internal effective magnetic field, as discussed above. The fact that Pd, Ir and Pt all exhibit a crossing field suggests a common mechanism of current-induced DW motion for these metals. To further explore the origin of the crossing field we introduce ultrathin 'dusting' layers of $\mathrm{Au}$ at the UL/Co interface in the magnetic stacks. For Au layers even just 1 or $2 \AA$ thick, as shown in Fig. 3a,b, the crossing field is markedly reduced and the role of the static DW pinning becomes more pronounced for all three ULs. The detailed dependence of $H_{\mathrm{CR}}, H_{\mathrm{fl}}$ and $\eta$ are shown in Fig. $3 c-e$ and discussed in more detail in the Supplementary Information. The DW velocity is decreased to zero for Au layers with thicknesses below $\sim 4 \AA$ in each case (Fig. 3f). Associated with changes in the DW velocity are significant changes in magnetization and magnetic anisotropy of the film stacks, as detailed in Fig. 3g,h. All the stacks remain perpendicularly magnetized for all $\mathrm{Au}$ layer thicknesses, although $K_{\text {eff }}$ drops substantially for Pt and Ir ULs, but the changes are much smaller for Pd ULs. Perhaps, most interestingly, for all three ULs the magnetization is much reduced, by a quantity $\delta M_{\mathrm{S}}$, with the introduction of the Au dusting layers, which we associate with the elimination of a PIM in the ULs that is induced by an exchange interaction with the neighbouring Co layer: $\delta M_{\mathrm{S}}$ is very large and ranges from 19 to $29 \%$ of $M_{\mathrm{S}}$ (see Table 1 ).

\section{Discussion}

The crossing field depends weakly on current density for ULs formed from Pd, Ir and Pt as well as these same ULs with Au dusting layers (when $H_{\mathrm{CR}} \geq 0$ ). This is the most important characteristic feature of the crossing field. Within the $1 \mathrm{D}$ model, since there is a contribution to $H_{\mathrm{CR}}$ from the $\mathrm{V}-\mathrm{STT}^{7,36}$ that increases linearly with $J$, we conclude that the V-STT contribution to $H_{\mathrm{CR}}$ is small. For the $\mathrm{Co} / \mathrm{Ni} / \mathrm{Co}$ stacks used here, this contribution, for $J \sim 2 \times 10^{8} \mathrm{~A} \mathrm{~cm}^{-2}$, is $\sim 140, \sim 240$ and $\sim 400$ Oe for Pd, Ir and Pt based stacks, respectively (see Supplementary Fig. 3), in each case decreasing the intrinsic crossing field, $H_{\mathrm{CR}}^{\mathrm{I}}$ (that is, $H_{\mathrm{CR}}$ in the absence of V-STT). Also, as anticipated within the 1D model (see Supplementary Fig. 2), as $J$ is increased, pinning can be more easily overcome leading to reduced $H_{\mathrm{fl}}$. Finally, $\eta$ is only weakly dependent on $J$ for Pt and $\mathrm{Pt} / \mathrm{Au}$ ULs, for which cases $H_{\mathrm{SHE}}$ is large compared with $H_{k}$, but increases with $J$ for Ir and Pd ULs where, by contrast, $H_{\mathrm{SHE}}$ is small compared with $H_{k}$ for small $J$. Thus, the dependence of $\eta$ on $J$ is consistent within the $1 \mathrm{D}$ model with a $J$ independent $\theta_{\mathrm{SHE}}$, as expected. Moreover, $\theta_{\mathrm{SHE}}$ is not much affected by the introduction of the Au dusting layers, thereby showing that the spin currents generated in the Pd, Ir and Pt ULs, via a SHE, can pass across the Au layers with little attenuation ${ }^{37}$ (see Supplementary Table 1 for the case of $\mathrm{Pt} / \mathrm{Au} / \mathrm{Co}$ ).

We finally discuss the origin of $H_{\mathrm{CR}}$ and its relationship with the magnetic properties of the different stacks. Assuming that the $J$-independent offset field is associated with a DMI, we postulate that $H_{\mathrm{CR}}$ will be proportional to the quantity $K K_{\mathrm{eff}}^{1 / 2} \delta M \mathrm{~s} / M \mathrm{~s}$. Our rationale is as follows: first, the DMI originates from the UL/Co interface that also determines the PMA in these stacks. Both the DMI and the PMA are, in a first approximation, proportional to the strength of spin-orbit coupling (SOC), and thus the DMI should be proportional to $K^{29,38,39}$. Second, since the DMI exchange energy density within the DW can be written as, $\sigma_{\mathrm{DMI}}=\mathbf{D} \cdot\left(\mathbf{m} \times \frac{\partial \mathbf{m}}{\partial x}\right)$, where $\mathbf{D}$ is a vector that reflects the strength and chirality of the $\mathrm{DMI}^{30}$, it follows that $\left\langle\sigma_{\mathrm{DMI}}\right\rangle \propto 1 / \Delta$, which, since $\Delta \propto K_{\mathrm{eff}}^{-1 / 2}$ means that $\left\langle\sigma_{\mathrm{DMI}}\right\rangle \propto K_{\mathrm{eff}}^{1 / 2}$. Third, we postulate that the DMI is also proportional to the PIM since the introduction of $\mathrm{Au}$ dusting layers eliminates the PIM but not the SHE. Figure $4 \mathrm{a}$ shows that $H_{\mathrm{CR}}$ increases in approximate proportion to the quantity $K K_{\text {eff }}^{1 / 2} \delta M s / M$ s consistent with our conjecture. Thus, we propose that the origin of the DMI is closely related to the PIM. In this regard, it is very interesting that the $\mathrm{Au} / \mathrm{Co}$ and $\mathrm{Pd} / \mathrm{Co}$ interfaces display very similar values of $K$ and $K_{\text {eff }}$ so that, as shown in Fig. $3 \mathrm{~h}$, there is very little change in $K_{\text {eff }}$ for Pd ULs when Au dusting layers are introduced. This is further supported by the similar dependence of $K_{\text {eff }}$ on the number of $\mathrm{Co} /$ $\mathrm{Ni}$ bilayers in $\mathrm{UL} / \mathrm{Co} /[\mathrm{Ni} / \mathrm{Co}]_{\mathrm{N}} / \mathrm{TaN}$ stacks as shown in Fig. $4 \mathrm{~b}$ for $\mathrm{Au}$ and $\mathrm{Pd}$ ULs, whereas there is a much stronger dependence of $K_{\text {eff }}$ for Ir and Pt ULs. Thus, one would anticipate a similar magnitude of the DMI for Au and Pd ULs that we do not find. 
The only distinction between Au and Pd ULs is the PIM, which we therefore argue is a critical ingredient in establishing significant values of DMI.

\section{Methods \\ Sample preparation. The films used in these experiments were deposited by magnetron sputter deposition at ambient temperature on $\mathrm{Si}(100)$ substrates covered with amorphous $\mathrm{SiO}_{2}$. A colourized optical micrograph of a typical device is shown in Fig. 1a. The wire in the central portion, where DW motion was imaged, is connected at both ends to wider regions that are used as bond pads for electrical connections. In most cases, we find that the PMA is significantly reduced at the wire bonds, such that DWs are nucleated near the bonds and can propagate in the wire under an external field (cartoon in Fig. 1a). Once a single DW is injected in the wire, the field is reduced to zero and DW motion is studied by applying a series of current pulses of length $t_{\mathrm{P}}$ to the device.}

Measurement of DW velocity. Kerr optical microscopy in differential mode is used to monitor the position of the DW along the nanowire in response to a series of current pulses (Fig. 1b). The sensitivity of this technique is sufficient to detect the motion of single DWs in nanowires as narrow as $\sim 100 \mathrm{~nm}$. Images are taken after a fixed number of current pulses chosen such that the DW has moved by a significant distance, typically $\sim 1-2 \mu \mathrm{m}$. The DW velocity is then determined by assuming that the DW moves only during the current pulses. We use a linear fit of the DW position versus the integrated current pulse length $t_{\mathrm{CP}}$, that is, the product of $t_{\mathrm{P}}$ and the number of pulses applied. In some cases, the DW may be pinned by a local defect until enough pulses are applied to dislodge it. In these cases, we fit only the portions of the curve in which the DW position depends linearly on $t_{\mathrm{CP}}$. The s.d. of the differential velocity, that is, the point by point derivative of the DW position versus $t_{\mathrm{CP}}$ curve, is used to determine an error bar. Note that we define the DW velocity to be positive (negative) when the DWs move along the current (against) flow.

DWs move over the entire length of the device at constant velocity, thus excluding driving mechanisms based on irreversible transformations of the DWs. $J$ is calculated from the device resistance by assuming uniform conduction in the metallic layers. DW motion takes place when $J$ exceeds a critical value $J_{\mathrm{C}}$. As shown in Fig. $1 \mathrm{~d}-\mathrm{g}, J_{\mathrm{C}}$ decreases significantly for longer current pulses as thermally activated processes become more important ${ }^{5}$. Above $J_{\mathrm{C}}$, the DW velocity increases rapidly with $J$. Motion at higher current densities is hindered by the random nucleation of reversed domains due to the combined action of STT, Joule heating and the Oersted field from the current.

Determination of magnetic anisotropy values. The magnetic anisotropy constants $K$ and $K_{\text {eff }}$ are determined by measuring the easy-axis and hard-axis magnetic hysteresis loops. When the easy axis component of magnetization $\left(M_{z}\right)$ is measured as a function of the magnetic field $H_{z}$ applied along the easy axis $(z)$, the hysteresis loop is rectangularly shaped (see Supplementary Fig. 4). At high enough $H_{z}, M_{z}$ equals the bulk saturation magnetization $M_{\mathrm{S}}$. In contrast, the hard axis component of magnetization $\left(M_{x}\right)$ increases linearly with $H_{x}$ when the field is applied along the hard axis $x$ (Supplementary Fig. 4). When $H_{x}$ equals the anisotropy field, $H_{\text {eff, }}, M_{x}=M_{\mathrm{S}}$, then, it follows that, $H_{\text {eff }}=2 K / M_{\mathrm{S}}-4 \pi M_{\mathrm{S}}$. Hence $K=K_{\text {eff }}+2 \pi M_{\mathrm{S}}^{2}$ and $K_{\text {eff }}=H_{\text {eff }} M_{\mathrm{S}} / 2$. Supplementary Fig. 5 shows typical easy and hard axis magnetization loops for samples with Ir, Pd, Pt and Au underlayers from which $K$ and $K_{\text {eff }}$ have been deduced.

\section{References}

1. Parkin, S. S. P., Hayashi, M. \& Thomas, L. Magnetic domain-wall racetrack memory. Science 320, 190-194 (2008).

2. Fukami, S. et al. Low-current perpendicular domain wall motion cell for scalable high-speed MRAM. Symposium on VLSI Technology Digest of Technical Papers 230-231, (2009).

3. Allwood, D. A. et al. Magnetic domain-wall logic. Science 309, 1688-1692 (2005).

4. Grollier, J. et al. Switching a spin valve back and forth by current-induced domain wall motion. Appl. Phys. Lett. 83, 509-511 (2003).

5. Yamaguchi, A. et al. Real-space observation of current-driven domain wall motion in submicron magnetic wires. Phys. Rev. Lett. 92, 077205 (2004).

6. Kläui, M. et al. Direct observation of domain-wall congurations transformed by spin currents. Phys. Rev. Lett. 95, 026601 (2005).

7. Thomas, L. et al. Oscillatory dependence of current-driven magnetic domain wall motion on current pulse length. Nature 443, 197-200 (2006).

8. Hayashi, M. et al. Current driven domain wall velocities exceeding the spin angular momentum transfer rate in permalloy nanowires. Phys. Rev. Lett. 98, 037204 (2007).

9. Koyama, T. et al. Observation of the intrinsic pinning of a magnetic domain wall in a ferromagnetic nanowire. Nat. Mater. 10, 194-197 (2011).

10. Berger, L. Exchange interaction between ferromagnetic domain wall and electric current in very thin metallic films. J. Appl. Phys. 55, 1954-1956 (1984).
11. Berger, L. Possible existence of a Josephson effect in ferromagnets. Phys. Rev. B 33, 1572-1578 (1986).

12. Slonczewski, J. Current driven excitation of magnetic multilayers. J. Magn. Magn. Mat. 159, L1-L7 (1996).

13. Miron, I. M. et al. Fast current-induced domain-wall motion controlled by the Rashba effect. Nat. Mater. 10, 419-423 (2011).

14. Ryu, K.-S., Thomas, L., Yang, S.-H. \& Parkin, S. S. P. Current induced tilting of domain walls in high velocity motion along perpendicularly magnetized micron-sized Co/Ni/Co racetracks. Appl. Phys. Express 5, 093006 (2012).

15. Ryu, K.-S., Thomas, L., Yang, S.-H. \& Parkin, S. Chiral spin torque at magnetic domain walls. Nat. Nano 8, 527-533 (2013).

16. Kim, K.-J. et al. Electric control of multiple domain walls in $\mathrm{Pt} / \mathrm{Co} / \mathrm{Pt}$ nanotrack with perpendicular magnetic anisotropy. Appl. Phys. Express 3, 083001 (2010).

17. Manchon, A. \& Zhang, S. Theory of spin torque due to spin-orbit coupling. Phys. Rev. B 79, 094422 (2009).

18. Miron, I. M. et al. Current-driven spin torque induced by the Rashba effect in a ferromagnetic metal layer. Nat. Mater. 9, 230-234 (2010).

19. Garello, K. et al. Symmetry and magnitude of spin-orbit torques in ferromagnetic heterostructures. Nat. Nano 8, 587-593 (2013).

20. Hirsch, J. E. Spin hall effect. Phys. Rev. Lett. 83, 1834-1837 (1999).

21. Zhang, S. Spin hall effect in the presence of spin diffusion. Phys. Rev. Lett. 85, 393 (2000).

22. Liu, L., Lee, O. J., Gudmundsen, T. J., Ralph, D. C. \& Buhrman, R. A. Current-induced switching of perpendicularly magnetized magnetic layers using spin torque from the spin hall effect. Phys. Rev. Lett. 109, 096602 (2012).

23. Thiaville, A., Rohart, S., Jue, E., Cros, V. \& Fert, A. Dynamics of Dzyaloshinskii domain walls in ultrathin magnetic films. Europhys. Lett. 100, 57002 (2012).

24. Khvalkovskiy, A. V. et al. Matching domain wall configuration and spin-orbit torques for very efficient domain-wall motion. Phys. Rev. B 87, 020402(R) (2013).

25. Emori, S., Bauer, U., Ahn, S.-M., Martinez, E. \& Beach, G. S. D. Current-driven dynamics of chiral ferromagnetic domain walls. Nat. Mater. 12, 611-616 (2013).

26. Dzyaloshinskii, I. E. Thermodynamic theory of weak ferromagnetism in antiferromagnetic substances. Sov. Phys. JETP 5, 1259-1272 (1957).

27. Dzyaloshinskii, I. E. Theory of helicoidal structures in antiferromagnets.1. Nonmetals. Sov. Phys. JETP 19, 960-971 (1964).

28. Moriya, T. Anisotropic superexchange interaction and weak ferromagnetism. Phys. Rev. 120, 91-98 (1960).

29. Bogdanov, A. N. \& Rößler, U. K. Chiral symmetry breaking in magnetic thin films and multilayers. Phys. Rev. Lett. 87, 037203 (2001).

30. Heide, M., Bihlmayer, G. \& Blügel, S. Dzyaloshinskii-Moriya interaction accounting for the orientation of magnetic domains in ultrathin films: Fe/W(110). Phys. Rev. B 78, 140403 (2008).

31. Vedmedenko, E. Y., Udvardi, L., Weinberger, P. \& Wiesendanger, R. Chiral magnetic ordering in two-dimensional ferromagnets with competing Dzyaloshinsky-Moriya interactions. Phys. Rev. B 75, 104431 (2007).

32. Mougin, A., Cormier, M., Adam, J. P., Metaxas, P. J. \& Ferré, J. Domain wall mobility, stability and Walker breakdown in magnetic nanowires. Europhys. Lett. 78, 57007 (2007).

33. Ando, K. \& Saitoh, E. Inverse spin-Hall effect in palladium at room temperature. J. Appl. Phys. 108, 113924-113925 (2010).

34. Morota, M. et al. Indication of intrinsic spin Hall effect in $4 \mathrm{~d}$ and $5 \mathrm{~d}$ transition metals. Phys. Rev. B 83, 174405 (2011).

35. Tanaka, T. et al. Intrinsic spin Hall effect and orbital Hall effect in $4 \mathrm{~d}$ and $5 \mathrm{~d}$ transition metals. Phys. Rev. B 77, 165117 (2008).

36. Thiaville, A., Nakatani, Y., Miltat, J. \& Suzuki, Y. Micromagnetic understanding of current-driven domain wall motion in patterned nanowires. Europhys. Lett. 69, 990-996 (2005).

37. Mihajlović, G., Pearson, J. E., Garcia, M. A., Bader, S. D. \& Hoffmann, A. Negative nonlocal resistance in mesoscopic gold hall bars: absence of the giant spin hall effect. Phys. Rev. Lett. 103, 166601-166604 (2009).

38. Nakajima, N. et al. Perpendicular magnetic anisotropy caused by interfacial hybridization via enhanced orbital moment in $\mathrm{Co} / \mathrm{Pt}$ multilayers: magnetic circular X-ray dichroism study. Phys. Rev. Lett. 81, 5229-5232 (1998).

39. Kim, K.-W., Lee, H.-W., Lee, K.-J. \& Stiles, M. D. Chirality from interfacial spin-orbit coupling effects in magnetic bilayers. Phys. Rev. Lett. 111, 216601 (2013).

\section{Acknowledgements}

K.-S.R. acknowledges financial support from the Max Planck Institute for Chemical Physics of Solids. We acknowledge partial support from the Army Research Office (contract \#: W911NF-13-1-0107).

\section{Author contributions}

S.S.P.P. conceived the study and designed the experiments. K.-S.R. performed the experiments and the data analysis. S.-H.Y. grew the film structures, carried out the sample fabrication and modelled the data. L.T. was involved in some of the 
experiments in the early stages of these studies. S.S.P.P., K.-S.R. and S.H.Y discussed the results and wrote the paper.

\section{Additional information}

Supplementary Information accompanies this paper at http://www.nature.com/

naturecommunications
Competing financial interests: The authors declare no competing financial interests.

Reprints and permission information is available online at http://npg.nature.com/ reprintsandpermissions/

How to cite this article: Ryu, K.-S. et al. Chiral spin torque arising from proximityinduced magnetization. Nat. Commun. 5:3910 doi: 10.1038/ncomms4910 (2014). 